\title{
A Study on Anti- Fuzzy HX Bi-Ideal of a HX Ring
}

\author{
R. Muthuraj ${ }^{1}$, N. Ramila Gandhi ${ }^{2}$ \\ ${ }^{1}$ Department of Mathematics, H.H.The Rajah’s College, Pudukkottai - 622 001, Tamilnadu, India. \\ ${ }^{2}$ Department of Mathematics, PSNA College of Engineering and Technology, Dindigul-624 622, Tamilnadu, India.
}

\begin{abstract}
In this paper, we introduce the notion of an anti-fuzzy $\mathrm{HX}$ bi-ideal of a $\mathrm{HX}$ ring. We discussed some related properties of an anti-fuzzy $\mathrm{HX}$ bi-ideal of a $\mathrm{HX}$ ring. We also discussed homomorphic image and pre-image of an anti-fuzzy $\mathrm{HX}$ bi-ideal of a $\mathrm{HX}$ ring.
\end{abstract}

Keywords: HX ring, fuzzy HX ring, HX bi- ideal, anti-fuzzy HX bi- ideal

\section{Introduction}

In 1965, Zadeh [16] introduced the concept of fuzzy sets and studied their properties. In 1967, Rosenfeld [13] defined the idea of fuzzy subgroups and gave some of its properties. Li Hong Xing [7] introduced the concept of HX group. In 1982 Wang-jin Liu [9] introduced the concept of fuzzy ring and fuzzy ideal. Kuroki [5] introduced the notion of fuzzy biideals in semi groups. In 1988 Professor Li Hong Xing [8] proposed the concept of HX ring and derived some of its properties, then Professor Zhong [1,2] gave the structures of HX ring on a class of ring. Lajos and Szasz [6] initiated the idea of bi-ideals in a ring. T.K.Mukherjee and M.K.Sen [11] fuzzified certain results on rings and ideals.

\section{Preliminaries}

In this section, we site the fundamental definitions that will be used in the sequel. Throughout this paper, $\mathrm{R}=(\mathrm{R},+, \cdot)$ is a Ring, $e$ is the additive identity element of $R$ and $x y$ we mean $\mathrm{x} \cdot \mathrm{y}$.

\section{Anti-fuzzy HX bi-ideal}

In this section we define anti fuzzy HX bi-ideal of a HX ring and discuss some of their properties.

\subsection{Definition}

Let $\mu$ be a fuzzy set defined on R. Let $\mathfrak{R} \subset 2^{R}-\{\phi\}$ be a HX ring. A fuzzy subset $\lambda_{\mu}$ of $\Re$ is said to be an anti-fuzzy HX biideal or anti-fuzzy bi-ideal induced by $\mu$ of a HX ring $\mathfrak{R}$ if the following conditions are satisfied. For all $\mathrm{A}, \mathrm{B}, \mathrm{C} \in \mathfrak{R}$,

i. $\lambda_{\mu}(\mathrm{A}-\mathrm{B}) \leq \max \left\{\lambda_{\mu}(\mathrm{A}), \lambda_{\mu}(\mathrm{B})\right\}$

ii. $\lambda_{\mu}(\mathrm{AB}) \leq \max \left\{\lambda_{\mu}(\mathrm{A}), \lambda_{\mu}(\mathrm{B})\right\}$

iii. $\lambda_{\mu}(\mathrm{ABC}) \leq \max \left\{\lambda_{\mu}(\mathrm{A}), \lambda_{\mu}(\mathrm{C})\right\}$

where, $\lambda_{\mu}(\mathrm{A})=\min \{\mu(\mathrm{x}) /$ for all $\mathrm{x} \in \mathrm{A} \subseteq \mathrm{R}\}$.

That is, Let $\mu$ be a fuzzy set defined on R. Let $\mathfrak{R} \subset 2^{R}-\{\phi\}$ be a HX ring. An anti-fuzzy HX subring $\lambda_{\mu}$ of $\Re$ is said to be an anti-fuzzy HX bi-ideal or an anti-fuzzy bi-ideal induced by $\mu$ of a HX ring $\mathfrak{R}$ if the following condition is satisfied. For all $\mathrm{A}, \mathrm{B}, \mathrm{C} \in \mathfrak{R}$,

$$
\lambda_{\mu}(\mathrm{ABC}) \leq \max \left\{\lambda_{\mu}(\mathrm{A}), \lambda_{\mu}(\mathrm{C})\right\}
$$
where, $\lambda_{\mu}(\mathrm{A})=\min \{\mu(\mathrm{x}) /$ for all $\mathrm{x} \in \mathrm{A} \subseteq \mathrm{R}\}$.

\subsection{Theorem}

If $\mu$ is an anti-fuzzy bi- ideal of a ring $\mathrm{R}$ then the fuzzy subset $\lambda_{\mu}$ is an anti-fuzzy HX bi- ideal of a HX ring $\mathfrak{R}$.

\section{Proof}

Let $\mu$ be an anti-fuzzy bi-ideal of $\mathrm{R}$.

i. $\max \left\{\lambda_{\mu}(\mathrm{A}), \lambda_{\mu}(\mathrm{B})\right\}=$

$\max \{\min \{\mu(\mathrm{x}) /$ for all $\mathrm{x} \in \mathrm{A} \subseteq \mathrm{R}\}$,

$=\max \left\{\mu\left(\mathrm{x}_{0}\right), \mu\left(\mathrm{y}_{0}\right)\right\}$ $\min \{\mu(\mathrm{y}) /$ for all $\mathrm{y} \in \mathrm{B} \subseteq \mathrm{R}\}\}$

$\geq \mu\left(x_{0}-y_{0}\right)$, since $\mu$ is an anti-fuzzy bi-ideal of $\mathrm{R} \geq$ $\min \{\mu(\mathrm{x}-\mathrm{y}) /$ for all $\mathrm{x}-\mathrm{y} \in \mathrm{A}-\mathrm{B} \subseteq \mathrm{R}\}$

$\geq \lambda_{\mu}(\mathrm{A}-\mathrm{B})$

$\lambda_{\mu}(\mathrm{A}-\mathrm{B}) \leq \max \left\{\lambda_{\mu}(\mathrm{A}), \lambda_{\mu}(\mathrm{B})\right\}$

ii. $\max \left\{\lambda_{\mu}(\mathrm{A}), \lambda_{\mu}(\mathrm{B})\right\}=$ $\max \{\min \{\mu(\mathrm{x}) /$ for all $\mathrm{x} \in \mathrm{A} \subseteq \mathrm{R}\}$,

$=\max \left\{\mu\left(\mathrm{x}_{0}\right), \mu\left(\mathrm{y}_{0}\right)\right\}$ $\min \{\mu(\mathrm{y}) /$ for all $\mathrm{y} \in \mathrm{B} \subseteq \mathrm{R}\}\}$

$\geq \mu\left(x_{0} y_{0}\right)$, since $\mu$ is an anti-fuzzy bi-ideal of $\mathrm{R}$

$\geq \min \{\mu(\mathrm{xy}) /$ for all $\mathrm{x}-\mathrm{y} \in \mathrm{A}-\mathrm{B} \subseteq \mathrm{R}\}$

$\geq \lambda_{\mu}(\mathrm{AB})$

$\lambda_{\mu}(\mathrm{AB}) \leq \max \left\{\lambda_{\mu}(\mathrm{A}), \lambda_{\mu}(\mathrm{B})\right\}$

iii.max $\left\{\lambda_{\mu}(\mathrm{A}), \lambda_{\mu}(\mathrm{C})\right\}$ $=\max \{\min \{\mu(\mathrm{x}) /$ for all $\mathrm{x} \in \mathrm{A} \subseteq \mathrm{R}\}$, $\min \{\mu(\mathrm{z}) /$ for all $\mathrm{z} \in \mathrm{C} \subseteq \mathrm{R}\}\}$

$=\max \left\{\mu\left(\mathrm{x}_{0}\right), \mu\left(\mathrm{z}_{0}\right)\right\}$

$\geq \mu\left(\mathrm{x}_{0} \mathrm{y}_{0} \mathrm{z}_{0}\right)$, since $\mu$ is an anti-fuzzy bi-ideal of $\mathrm{R}$

$\geq \min \{\mu(\mathrm{xyz}) /$ for all $\mathrm{xyz} \in \mathrm{ABC} \subseteq \mathrm{R}$

$\geq \lambda_{\mu}(\mathrm{ABC})$

$\lambda_{\mu}(\mathrm{ABC}) \leq \max \left\{\lambda_{\mu}(\mathrm{A}), \lambda_{\mu}(\mathrm{C})\right\}$

Hence, $\lambda_{\mu}$ is an anti-fuzzy HX bi-ideal of a HX ring $\mathfrak{R}$.

\subsection{Remark}

i. If $\mu$ is not an anti-fuzzy bi-ideal of $\mathrm{R}$ then the fuzzy subset $\lambda_{\mu}$ of $\Re$ is an anti-fuzzy HX bi-ideal of $\mathfrak{R}$, provided $|\mathrm{X}|$ $\geq 2$ for all $X \in \mathfrak{R}$.

ii. If $\mu$ is a fuzzy subset of a ring $R$ and $\lambda_{\mu}$ be an anti-fuzzy HX bi-ideal on $\Re$, such that

$\lambda_{\mu}(\mathrm{A})=\min \{\mu(\mathrm{x}) /$ for all $\mathrm{x} \in \mathrm{A} \subseteq \mathrm{R}\}$, then $\mu$ may or may not be an anti-fuzzy bi-ideal of $\mathrm{R}$. 


\section{International Journal of Science and Research (IJSR) \\ ISSN (Online): 2319-7064 \\ Index Copernicus Value (2013): 6.14 | Impact Factor (2015): 6.391}

\subsection{Theorem}

Let $\mu$ be a fuzzy subset on R. Let $\Re \subset 2^{R}-\{\phi\}$ be a HX ring. If $\lambda_{\mu}$ is an anti-fuzzy HX right ideal of a HX ring $\mathfrak{R}$ then $\lambda_{\mu}$ is an anti-fuzzy $\mathrm{HX}$ bi-ideal of a HX ring $\mathfrak{R}$.

\section{Proof}

Let $\lambda_{\mu}$ be an anti-fuzzy HX right ideal of a HX ring $\mathfrak{R}$.

Then for all $\mathrm{A}, \mathrm{B} \in \mathfrak{R}$.

$$
\begin{aligned}
& \lambda_{\mu}(\mathrm{A}-\mathrm{B}) \leq \max \left\{\lambda_{\mu}(\mathrm{A}), \lambda_{\mu}(\mathrm{B})\right\} \\
& \lambda_{\mu}(\mathrm{AB}) \leq \lambda_{\mu}(\mathrm{A}) .
\end{aligned}
$$

Therefore, $\quad \lambda_{\mu}(\mathrm{AB}) \leq \max \left\{\lambda_{\mu}(\mathrm{A}), \lambda_{\mu}(\mathrm{B})\right\}$

Let $\mathrm{A}, \mathrm{B}, \mathrm{C} \in \mathfrak{R}$,

$$
\begin{aligned}
\lambda_{\mu}(\mathrm{ABC})= & \lambda_{\mu}(\mathrm{A}(\mathrm{BC})) \\
& \leq \lambda_{\mu}(\mathrm{A}), \\
& \leq \max \left\{\lambda_{\mu}(\mathrm{A}), \lambda_{\mu}(\mathrm{C})\right\} . \\
\lambda_{\mu}(\mathrm{ABC}) & \leq \max \left\{\lambda_{\mu}(\mathrm{A}), \lambda_{\mu}(\mathrm{C})\right\} .
\end{aligned}
$$

Hence, $\lambda_{\mu}$ is an anti-fuzzy $\mathrm{HX}$ bi-ideal of a HX ring $\mathfrak{R}$.

\subsection{Theorem}

Let $\mu$ be a fuzzy subset on R. Let $\Re \subset 2^{R}-\{\phi\}$ be a HX ring. If $\lambda_{\mu}$ is an anti-fuzzy HX left ideal of a HX ring $\mathfrak{R}$ then $\lambda_{\mu}$ is an anti-fuzzy HX bi-ideal of a HX ring $\Re$.

\section{Proof}

Let $\lambda_{\mu}$ be an anti-fuzzy HX left ideal of a HX ring $\Re$.

Then for all $\mathrm{A}, \mathrm{B} \in \mathfrak{R}$.

$$
\begin{aligned}
\lambda_{\mu}(\mathrm{A}-\mathrm{B}) & \leq \max \left\{\lambda_{\mu}(\mathrm{A}), \lambda_{\mu}(\mathrm{B})\right\} \\
\lambda_{\mu}(\mathrm{AB}) & \leq \lambda_{\mu}(\mathrm{B}) .
\end{aligned}
$$

Therefore, $\quad \lambda_{\mu}(\mathrm{AB}) \leq \max \left\{\lambda_{\mu}(\mathrm{A}), \lambda_{\mu}(\mathrm{B})\right\}$ Let $\mathrm{A}, \mathrm{B}, \mathrm{C} \in \mathfrak{R}$,

$$
\begin{aligned}
& \lambda_{\mu}(\mathrm{ABC})= \lambda_{\mu}((\mathrm{AB}) \mathrm{C}) \\
& \leq \lambda_{\mu}(\mathrm{C}), \\
& \leq \max \left\{\lambda_{\mu}(\mathrm{A}), \lambda_{\mu}(\mathrm{C})\right\} . \\
& \lambda_{\mu}(\mathrm{ABC}) \leq \max \left\{\lambda_{\mu}(\mathrm{A}), \lambda_{\mu}(\mathrm{C})\right\} .
\end{aligned}
$$

Hence, $\lambda_{\mu}$ is an anti-fuzzy $\mathrm{HX}$ bi-ideal of a HX ring $\mathfrak{R}$.

\subsection{Remark}

Every anti-fuzzy (right or left) HX ideal of a HX ring $\mathfrak{R}$ is an anti-fuzzy HX bi-ideal of a HX ring $\mathfrak{R}$.

\subsection{Theorem}

Let $\mu$ and $\eta$ be two fuzzy sets defined on R. Let $\lambda_{\mu}$ and $\gamma_{\eta}$ be any two anti-fuzzy HX bi-ideals of a HX ring $\mathfrak{R}$ then $\lambda_{\mu} \cap \gamma_{\eta}$ is also anti-fuzzy $\mathrm{HX}$ bi-ideal of a HX ring $\mathfrak{R}$.

\section{Proof}

Let $\mathrm{A}, \mathrm{B}, \mathrm{C} \in \mathfrak{R}$

$$
\begin{aligned}
& \text { i. } \quad\left(\lambda_{\mu} \cap \gamma_{\eta}\right)(\mathrm{A}-\mathrm{B})=\min \left\{\lambda_{\mu}(\mathrm{A}-\mathrm{B}), \gamma_{\eta}(\mathrm{A}-\mathrm{B})\right\} \\
& \leq \min \left\{\max \left\{\lambda_{\mu}(\mathrm{A}), \lambda_{\mu}(\mathrm{B})\right\}, \max \left\{\gamma_{\eta}(\mathrm{A}), \gamma_{\eta}(\mathrm{B})\right\}\right\} \\
& \leq \max \left\{\min \left\{\lambda_{\mu}(\mathrm{A}), \gamma_{\eta}(\mathrm{A})\right\}, \min \left\{\lambda_{\mu}(\mathrm{B}), \gamma_{\eta}(\mathrm{B})\right\}\right\} \\
& \leq \max \left\{\left(\lambda_{\mu} \cap \gamma_{\eta}\right)(\mathrm{A}),\left(\lambda_{\mu} \cap \gamma_{\eta}\right)(\mathrm{B})\right\} .
\end{aligned}
$$

\section{Therefore}

$$
\begin{gathered}
\left(\lambda_{\mu} \cap \gamma_{\eta}\right)(\mathrm{A}-\mathrm{B}) \leq \max \left\{\left(\lambda_{\mu} \cap \gamma_{\eta}\right)(\mathrm{A}),\left(\lambda_{\mu} \cap \gamma_{\eta}\right)(\mathrm{B})\right\} . \\
\text { ii. } \quad\left(\lambda_{\mu} \cap \gamma_{\eta}\right)(\mathrm{AB})=\min \left\{\lambda_{\mu}(\mathrm{AB}), \gamma_{\eta}(\mathrm{AB})\right\}
\end{gathered}
$$

$\leq \min \left\{\max \left\{\lambda_{\mu}(\mathrm{A}), \lambda_{\mu}(\mathrm{B})\right\}, \max \left\{\gamma_{\eta}(\mathrm{A}), \gamma_{\eta}(\mathrm{B})\right\}\right\}$

$\leq \max \left\{\min \left\{\lambda_{\mu}(\mathrm{A}), \gamma_{\eta}(\mathrm{A})\right\}, \min \left\{\lambda_{\mu}(\mathrm{B}), \gamma_{\eta}(\mathrm{B})\right\}\right\}$

$\geq \max \left\{\left(\lambda_{\mu} \cap \gamma_{\eta}\right)(\mathrm{A}),\left(\lambda_{\mu} \cap \gamma_{\eta}\right)(\mathrm{B})\right\}$

Therefore,

$$
\begin{aligned}
& \left(\lambda_{\mu} \cap \gamma_{\eta}\right)(\mathrm{AB}) \geq \max \left\{\left(\lambda_{\mu} \cap \gamma_{\eta}\right)(\mathrm{A}),\left(\lambda_{\mu} \cap \gamma_{\eta}\right)(\mathrm{B})\right\} . \\
& \text { iii. } \quad\left(\lambda_{\mu} \cap \gamma_{\eta}\right)(\mathrm{ABC})=\min \left\{\lambda_{\mu}(\mathrm{ABC}), \gamma_{\eta}(\mathrm{ABC})\right\} \\
& \leq \min \left\{\max \left\{\lambda_{\mu}(\mathrm{A}), \lambda_{\mu}(\mathrm{C})\right\}, \max \left\{\gamma_{\eta}(\mathrm{A}), \gamma_{\eta}(\mathrm{C})\right\}\right\} \\
& \leq \max \left\{\min \left\{\lambda_{\mu}(\mathrm{A}), \gamma_{\eta}(\mathrm{A})\right\}, \min \left\{\lambda_{\mu}(\mathrm{C}), \gamma_{\eta}(\mathrm{C})\right\}\right\} \\
& \quad \leq \max \left\{\left(\lambda_{\mu} \cap \gamma_{\eta}\right)(\mathrm{A}),\left(\lambda_{\mu} \cap \gamma_{\eta}\right)(\mathrm{C})\right\}
\end{aligned}
$$

Therefore,

$$
\left(\lambda_{\mu} \cap \gamma_{\eta}\right)(\mathrm{ABC}) \leq \max \left\{\left(\lambda_{\mu} \cap \gamma_{\eta}\right)(\mathrm{A}),\left(\lambda_{\mu} \cap \gamma_{\eta}\right)(\mathrm{C})\right\} .
$$

Hence, $\lambda_{\mu} \cap \gamma_{\eta}$ is an anti-fuzzy HX bi-ideal of a HX ring $\mathfrak{R}$.

\subsection{Theorem}

Let $\lambda_{\mu}$ be an anti-fuzzy HX (right or left) ideal and $\gamma_{\eta}$ be an anti-fuzzy HX (right or left) ideal then $\lambda_{\mu} \cap \gamma_{\eta}$ is an antifuzzy HX bi-ideal of $\mathfrak{R}$.

\section{Proof}

It is clear.

\subsection{Theorem}

Let $\mu$ and $\eta$ be any two fuzzy sets of R. Let $\Re \subset 2^{R}-\{\phi\}$ be a HX ring and if $\lambda_{\mu}$ and $\gamma_{\eta}$ are any two anti-fuzzy HX biideals of $\mathfrak{R}$ then $\left(\lambda_{\mu} \cup \gamma_{\eta}\right)$ is also an anti-fuzzy HX biideal of a HX ring $\Re$.

\section{Proof}

It is clear.

\subsection{Definition}

Let $\mu$ and $\eta$ be fuzzy subsets of the rings $R_{1}$ and $R_{2}$ respectively. Let $\lambda_{\mu}$ and $\gamma_{\eta}$ be two anti-fuzzy HX bi-ideals of the HX rings $\mathfrak{R}_{1}$ and $\mathfrak{R}_{2}$ then the cartesian anti-product of $\lambda_{\mu}$ and $\gamma_{\eta}$ is defined as $\left(\lambda_{\mu} \times \gamma_{\eta}\right)(\mathrm{A}, \mathrm{B})=\max \left\{\lambda_{\mu}(\mathrm{A}), \gamma_{\eta}(\mathrm{B})\right\}$ for every $(\mathrm{A}, \mathrm{B}) \in \mathfrak{R}_{1} \times \mathfrak{R}_{2}$.

\subsection{Theorem}

If $\lambda_{\mu}$ and $\gamma_{\eta}$ are any two anti-fuzzy HX bi-ideals of HX rings $\mathfrak{R}_{1}$ and $\mathfrak{R}_{2}$ then $\lambda_{\mu} \times \gamma_{\eta}$ is also an anti-fuzzy HX bi-ideal of $\mathfrak{R}_{1} \times \mathfrak{R}_{2}$.

\section{Proof}

Let $\lambda_{\mu}$ and $\gamma_{\eta}$ be any two anti-fuzzy HX bi-ideals of $\mathfrak{R}_{1}$ and $\mathfrak{R}_{2}$ respectively

Let $\mathrm{A}, \mathrm{B}, \mathrm{C} \in \mathfrak{R}_{1} \times \mathfrak{R}_{2}$ where $\mathrm{A}=(\mathrm{D}, \mathrm{E}), \mathrm{B}=(\mathrm{F}, \mathrm{G}), \mathrm{C}=(\mathrm{H}, \mathrm{I})$.

$$
\text { i. } \begin{aligned}
\left(\lambda_{\mu} \times \gamma_{\eta}\right)(\mathrm{A}-\mathrm{B})=\left(\lambda_{\mu} \times \gamma_{\eta}\right)((\mathrm{D}, \mathrm{E})-(\mathrm{F}, \mathrm{G})) \\
=\left(\lambda_{\mu} \times \gamma_{\eta}\right)(\mathrm{D}-\mathrm{F}, \mathrm{E}-\mathrm{G}) \\
=\max \left\{\lambda_{\mu}(\mathrm{D}-\mathrm{F}), \gamma_{\eta}(\mathrm{E}-\mathrm{G})\right\} \\
\leq \max \left\{\max \left\{\lambda_{\mu}(\mathrm{D}), \lambda_{\mu}(\mathrm{F})\right\},\right. \\
\left.\max \left\{\gamma_{\eta}(\mathrm{E}), \gamma_{\eta}(\mathrm{G})\right\}\right\} \\
\leq \max \left\{\max \left\{\lambda_{\mu}(\mathrm{C}), \gamma_{\eta}(\mathrm{E})\right\},\right. \\
\left.\max \left\{\lambda_{\mu}(\mathrm{F}), \gamma_{\eta}(\mathrm{G})\right\}\right\} \\
\leq \max \left\{\left(\lambda_{\mu} \times \gamma_{\eta}\right)(\mathrm{C}, \mathrm{E}),\left(\lambda_{\mu} \times \gamma_{\eta}\right)(\mathrm{F}, \mathrm{G})\right\}
\end{aligned}
$$




\section{International Journal of Science and Research (IJSR) \\ ISSN (Online): 2319-7064}

Index Copernicus Value (2013): 6.14 | Impact Factor (2015): 6.391

$=\max \left\{\left(\lambda_{\mu} \times \gamma_{\eta}\right)(\mathrm{A}),\left(\lambda_{\mu} \times \gamma_{\eta}\right)(\mathrm{B})\right\} \quad\left(\lambda_{\mu} \times \gamma_{\eta}\right)$

$(\mathrm{A}-\mathrm{B}) \leq \max \left\{\left(\lambda_{\mu} \times \gamma_{\eta}\right)(\mathrm{A}),\left(\lambda_{\mu} \times \gamma_{\eta}\right)(\mathrm{B})\right\}$.

ii. $\left(\lambda_{\mu} \times \gamma_{\eta}\right)(\mathrm{AB})=\left(\lambda_{\mu} \times \gamma_{\eta}\right)((\mathrm{D}, \mathrm{E})(\mathrm{F}, \mathrm{G}))$

$$
=\left(\lambda_{\mu} \times \gamma_{\eta}\right)(\mathrm{DF}, \mathrm{EG})
$$

$=\max \left\{\lambda_{\mu}(\mathrm{DF}), \gamma_{\eta}(\mathrm{EG})\right\}$

$\leq \max \left\{\max \left\{\lambda_{\mu}(\mathrm{D}), \lambda_{\mu}(\mathrm{F})\right\}\right.$, $\left.\max \left\{\gamma_{\eta}(\mathrm{E}), \gamma_{\eta}(\mathrm{G})\right\}\right\}$

$=\max \left\{\max \left\{\lambda_{\mu}(\mathrm{D}), \gamma_{\eta}(\mathrm{E})\right\}\right.$, $\left.\max \left\{\lambda_{\mu}(\mathrm{F}), \gamma_{\eta}(\mathrm{G})\right\}\right\}$

$\leq \max \left\{\left(\lambda_{\mu} \times \gamma_{\eta}\right)(\mathrm{D}, \mathrm{E}),\left(\lambda_{\mu} \times \gamma_{\eta}\right)(\mathrm{F}, \mathrm{G})\right\}$

$=\max \left\{\left(\lambda_{\mu} \times \gamma_{\eta}\right)(\mathrm{A}),\left(\lambda_{\mu} \times \gamma_{\eta}\right)(\mathrm{B})\right\}$

$\left(\lambda_{\mu} \times \gamma_{\eta}\right)(\mathrm{AB}) \leq \max \left\{\left(\lambda_{\mu} \times \gamma_{\eta}\right)(\mathrm{A}),\left(\lambda_{\mu} \times \gamma_{\eta}\right)(\mathrm{B})\right\}$

iii. $\left(\lambda_{\mu} \times \gamma_{\eta}\right)(\mathrm{ABC})=\left(\lambda_{\mu} \times \gamma_{\eta}\right)(\mathrm{DFH}, \mathrm{EGI})$

$=\max \left\{\lambda_{\mu}(\mathrm{DFH}), \gamma_{\eta}(\mathrm{EGI})\right\}$

$\leq \max \left\{\max \left\{\lambda_{\mu}(\mathrm{D}), \lambda_{\mu}(\mathrm{H})\right\}\right.$ , $\left.\max \left\{\gamma_{\eta}(\mathrm{E}), \gamma_{\eta}(\mathrm{I})\right\}\right\}$

$=\max \left\{\max \left\{\lambda_{\mu}(\mathrm{D}), \gamma_{\eta}(\mathrm{E})\right\}\right.$, $\left.\max \left\{\lambda_{\mu}(\mathrm{H}), \gamma_{\eta}(\mathrm{I})\right\}\right\}$

$\leq \max \left\{\left(\lambda_{\mu} \times \gamma_{\eta}\right)(\mathrm{D}, \mathrm{E}),\left(\lambda_{\mu} \times \gamma_{\eta}\right)(\mathrm{H}, \mathrm{I})\right\}$

$\left(\lambda_{\mu} \times \gamma_{\eta}\right)(\mathrm{ABC}) \leq \max \left\{\left(\lambda_{\mu} \times \gamma_{\eta}\right)(\mathrm{A}),\left(\lambda_{\mu} \times \gamma_{\eta}\right)(\mathrm{C})\right\}$

Hence, $\lambda_{\mu} \times \gamma_{\eta}$ is an anti-fuzzy HX bi-ideal of $\mathfrak{R}_{1} \times \mathfrak{R}_{2}$.

\subsection{Theorem}

Let $\mu$ be a fuzzy set defined on R. Let $\lambda^{\mu}$ is a fuzzy HX biideal of $\Re$ if and only if $\left(\lambda^{\mu}\right)^{\mathrm{c}}$ is an anti-fuzzy HX bi-ideal of $\mathfrak{R}$.

\section{Proof}

Let $\lambda^{\mu}$ be a fuzzy HX bi-ideal of $\Re$.

Let $\mathrm{A}, \mathrm{B} \in \mathfrak{R}$

$$
\text { i. } \lambda^{\mu}(\mathrm{A}-\mathrm{B}) \geq \min \left\{\lambda^{\mu}(\mathrm{A}), \lambda^{\mu}(\mathrm{B})\right\}
$$

$\Leftrightarrow 1-\left(\lambda^{\mu}\right)^{\mathrm{c}}(\mathrm{A}-\mathrm{B}) \geq \min \left\{\left(1-\left(\lambda^{\mu}\right)^{\mathrm{c}}(\mathrm{A})\right),\left(1-\left(\lambda^{\mu}\right)^{\mathrm{c}}(\mathrm{B})\right)\right\}$

$\Leftrightarrow \quad\left(\lambda^{\mu}\right)^{\mathrm{c}}(\mathrm{A}-\mathrm{B}) \leq 1-\min \left\{\left(1-\left(\lambda^{\mu}\right)^{\mathrm{c}}(\mathrm{A})\right),\left(1-\left(\lambda^{\mu}\right)^{\mathrm{c}}(\mathrm{B})\right)\right\}$

$\Leftrightarrow \quad\left(\lambda^{\mu}\right)^{\mathrm{c}}(\mathrm{A}-\mathrm{B}) \leq \max \left\{\left(\lambda^{\mu}\right)^{\mathrm{c}}(\mathrm{A}),\left(\lambda^{\mu}\right)^{\mathrm{c}}(\mathrm{B})\right\}$

ii. $\lambda^{\mu}(\mathrm{AB}) \geq \min \left\{\lambda^{\mu}(\mathrm{A}), \lambda^{\mu}(\mathrm{B})\right\}$

$\Leftrightarrow 1-\left(\lambda^{\mu}\right)^{\mathrm{c}}(\mathrm{AB}) \geq \min \left\{\left(1-\left(\lambda^{\mu}\right)^{\mathrm{c}}(\mathrm{A})\right),\left(1-\left(\lambda^{\mu}\right)^{\mathrm{c}}(\mathrm{B})\right)\right\}$

$\Leftrightarrow \quad\left(\lambda^{\mu}\right)^{\mathrm{c}}(\mathrm{AB}) \leq 1-\min \left\{\left(1-\left(\lambda^{\mu}\right)^{\mathrm{c}}(\mathrm{A})\right),\left(1-\left(\lambda^{\mu}\right)^{\mathrm{c}}(\mathrm{B})\right)\right\}$

$\Leftrightarrow\left(\lambda^{\mu}\right)^{\mathrm{c}}(\mathrm{AB}) \leq \max \left\{\left(\lambda^{\mu}\right)^{\mathrm{c}}(\mathrm{A}),\left(\lambda^{\mu}\right)^{\mathrm{c}}(\mathrm{B})\right\}$

iii. $\lambda^{\mu}(\mathrm{ABC}) \geq \min \left\{\lambda^{\mu}(\mathrm{A}), \lambda^{\mu}(\mathrm{C})\right\}$

$\Leftrightarrow 1-\left(\lambda^{\mu}\right)^{\mathrm{c}}(\mathrm{ABC}) \geq \min \left\{\left(1-\left(\lambda^{\mu}\right)^{\mathrm{c}}(\mathrm{A})\right),\left(1-\left(\lambda^{\mu}\right)^{\mathrm{c}}(\mathrm{C})\right)\right\}$

$\Leftrightarrow\left(\lambda^{\mu}\right)^{\mathrm{c}}(\mathrm{ABC}) \leq 1-\min \left\{\left(1-\left(\lambda^{\mu}\right)^{\mathrm{c}}(\mathrm{A})\right),\left(1-\left(\lambda^{\mu}\right)^{\mathrm{c}}(\mathrm{C})\right)\right\}$

$\Leftrightarrow\left(\lambda^{\mu}\right)^{\mathrm{c}}(\mathrm{ABC}) \leq \max \left\{\left(\lambda^{\mu}\right)^{\mathrm{c}}(\mathrm{A}),\left(\lambda^{\mu}\right)^{\mathrm{c}}(\mathrm{C})\right\}$

Hence, $\left(\lambda^{\mu}\right)^{c}$ is an anti-fuzzy HX bi-ideal on $\mathfrak{R}$.

\section{Homomorphism and anti homomorphism of an anti-fuzzy $\mathrm{HX}$ bi-ideal of a $\mathrm{HX}$ ring $\mathfrak{R}$}

In this section, we discuss the properties of fuzzy HX bi-ideal of a HX ring $\mathfrak{R}$ under homomorphism and anti homomorphism.

\subsection{Definition}

Let $\mathrm{R}_{1}$ and $\mathrm{R}_{2}$ be any two rings. Let $\mathfrak{R}_{1} \subset 2^{\mathrm{R} 1}-\{\phi\}$ and $\mathfrak{R}_{2} \subset$ $2^{\mathrm{R} 2}-\{\phi\}$ be any two $\mathrm{HX}$ rings defined on $\mathrm{R}_{1}$ and $\mathrm{R}_{2}$ respectively. Let $\mu$ and $\alpha$ be any two fuzzy subsets in $R_{1}$ and $\mathrm{R}_{2}$ respectively. Let $\lambda_{\mu}$ and $\eta_{\alpha}$ be anti-fuzzy $\mathrm{HX}$ bi-ideals defined on $\mathfrak{R}_{1}$ and $\mathfrak{R}_{2}$ respectively induced by $\mu$ and $\alpha$. Let $\mathrm{f}$ :
$\mathfrak{R}_{1} \rightarrow \mathfrak{R}_{2}$ be a mapping then the anti-image of $\lambda_{\mu}$ denoted as $\mathrm{f}\left(\lambda_{\mu}\right)$ is a fuzzy subset of $\mathfrak{R}_{2}$ defined as for each $U \in \mathfrak{R}_{2}$, (f $\left.\left(\lambda_{\mu}\right)\right)(\mathrm{U})=$

$$
\left\{\begin{array}{cc}
\inf \left\{\lambda_{\mu}(\mathrm{X}): \mathrm{X} \in \mathrm{f}^{-1}(\mathrm{U})\right\}, & \text { if } \mathrm{f}^{-1}(\mathrm{U}) \neq \phi \\
1, & \text { otherwise }
\end{array}\right.
$$

Also the anti-pre-image of $\eta^{\alpha}$ denoted as $\mathrm{f}^{-1}\left(\eta_{\alpha}\right)$ under $\mathrm{f}$ is a fuzzy subset of $\mathfrak{R}_{1}$ defined as for each $\mathrm{X} \in \mathfrak{R}_{1}$, $\left(f^{-1}\left(\eta_{\alpha}\right)\right)(X)=\eta_{\alpha}(f(X))$.

\subsection{Theorem}

Let $\Re_{1}$ and $\Re_{2}$ be any two HX rings on the rings $R_{1}$ and $R_{2}$ respectively. Let $\mathrm{f}: \mathfrak{R}_{1} \rightarrow \mathfrak{R}_{2}$ be a homomorphism onto HX rings. Let $\lambda_{\mu}$ be an anti-fuzzy HX bi-ideal of $\Re_{1}$ then $f\left(\lambda_{\mu}\right)$ is an anti-fuzzy HX bi-ideal of $\mathfrak{R}_{2}$, if $\lambda_{\mu}$ has an infimum property and $\lambda_{\mu}$ is f-invariant.

\section{Proof}

Let $\mu$ be a fuzzy subset of $R_{1}$ and $\lambda_{\mu}$ is an anti-fuzzy HX bi-ideal of $\Re_{1}$.

There exist $X, Y, Z \in \mathfrak{R}_{1}$ such that $\mathrm{f}(\mathrm{X}), \mathrm{f}(\mathrm{Y}), \mathrm{f}(\mathrm{Z}) \in \mathfrak{R}_{2}$,

i. $\left(f\left(\lambda_{\mu}\right)\right)(f(X)-f(Y))=\left(f\left(\lambda_{\mu}\right)\right)(f(X-Y))$,

$$
\begin{aligned}
& \lambda_{\mu}(\mathrm{X}-\mathrm{Y}) \\
\leq & \max \left\{\lambda_{\mu}(\mathrm{X}), \lambda_{\mu}(\mathrm{Y})\right\} \\
= & \max \left\{\left(\mathrm{f}\left(\lambda_{\mu}\right)\right)(\mathrm{f}(\mathrm{X})),\left(\mathrm{f}\left(\lambda_{\mu}\right)\right)(\mathrm{f}(\mathrm{Y}))\right\}
\end{aligned}
$$

Therefore, $\left(\mathrm{f}\left(\lambda_{\mu}\right)\right)(\mathrm{f}(\mathrm{X})-\mathrm{f}(\mathrm{Y})) \leq$ $\max \left\{\left(\mathrm{f}\left(\lambda_{\mu}\right)\right)(\mathrm{f}(\mathrm{X})),\left(\mathrm{f}\left(\lambda_{\mu}\right)\right)(\mathrm{f}(\mathrm{Y}))\right\}$.

ii. $\left(\mathrm{f}\left(\lambda_{\mu}\right)\right)(\mathrm{f}(\mathrm{X}) \mathrm{f}(\mathrm{Y}))=\left(\mathrm{f}\left(\lambda_{\mu}\right)\right)(\mathrm{f}(\mathrm{XY}))$,

$$
\begin{aligned}
= & \lambda_{\mu}(\mathrm{XY}) \\
\leq & \max \left\{\lambda_{\mu}(\mathrm{X}), \lambda_{\mu}(\mathrm{Y})\right\}
\end{aligned}
$$$$
=\max \left\{\left(\mathrm{f}\left(\lambda_{\mu}\right)\right)(\mathrm{f}(\mathrm{X})),\left(\mathrm{f}\left(\lambda_{\mu}\right)\right)(\mathrm{f}(\mathrm{Y}))\right\}
$$

Therefore, $\left(\mathrm{f}\left(\lambda_{\mu}\right)\right)(\mathrm{f}(\mathrm{X}) \mathrm{f}(\mathrm{Y}))$

iii. $\left(\mathrm{f}\left(\lambda_{\mu}\right)\right)(\mathrm{f}(\mathrm{X}) \mathrm{f}(\mathrm{Y}) \mathrm{f}(\mathrm{Z}))=\left(\mathrm{f}\left(\lambda_{\mu}\right)\right)(\mathrm{f}(\mathrm{XYZ}))$$$
\leq \max \left\{\left(\mathrm{f}\left(\lambda_{\mu}\right)\right)\left(\mathrm{f}(\mathrm{X}),\left(\mathrm{f}\left(\lambda_{\mu}\right)\right)(\mathrm{f}(\mathrm{Y}))\right\} .\right.
$$

$$
\begin{aligned}
& =\lambda_{\mu}(\mathrm{XYZ}) \\
& \leq \max \left\{\lambda_{\mu}(\mathrm{X}), \lambda_{\mu}(\mathrm{Z})\right\}
\end{aligned}
$$$$
=\max \left\{\left(\mathrm{f}\left(\lambda_{\mu}\right)\right)(\mathrm{f}(\mathrm{X})),\left(\mathrm{f}\left(\lambda_{\mu}\right)\right)(\mathrm{f}(\mathrm{Z}))\right\}
$$

$\left(\mathrm{f}\left(\lambda_{\mu}\right)\right)(\mathrm{f}(\mathrm{X}) \mathrm{f}(\mathrm{Y}) \mathrm{f}(\mathrm{Z})) \leq \max \left\{\left(\mathrm{f}\left(\lambda_{\mu}\right)\right)(\mathrm{f}(\mathrm{X})),\left(\mathrm{f}\left(\lambda_{\mu}\right)\right)(\mathrm{f}(\mathrm{Z}))\right\}$

Hence, $\left(\mathrm{f}\left(\lambda_{\mu}\right)\right)$ is an anti-fuzzy HX bi- ideal on $\mathfrak{R}_{2}$.

\subsection{Theorem}

Let $\mathfrak{R}_{1}$ and $\mathfrak{R}_{2}$ be any two $\mathrm{HX}$ rings on $\mathrm{R}_{1}$ and $\mathrm{R}_{2}$ respectively. Let f : $\mathfrak{R}_{1} \rightarrow \mathfrak{R}_{2}$ be a homomorphism on HX rings. Let $\eta_{\alpha}$ be an anti-fuzzy HX bi-ideal of $\mathfrak{R}_{2}$ then $\mathrm{f}^{-1}\left(\eta_{\alpha}\right)$ is an anti-fuzzy $\mathrm{HX}$ bi- ideal of $\mathfrak{R}_{1}$.

\section{Proof}

Let $\mu$ be a fuzzy subset of $R_{1}$ and $\eta_{\alpha}$ is an anti-fuzzy HX biideal of $\Re_{1}$.

There exist $X, Y, Z \in \mathfrak{R}_{1}$ such that $\mathrm{f}(\mathrm{X}), \mathrm{f}(\mathrm{Y}), \mathrm{f}(\mathrm{Z}) \in \mathfrak{R}_{2}$,

i. $\left(\mathrm{f}^{-1}\left(\eta_{\alpha}\right)\right)(\mathrm{X}-\mathrm{Y})=\eta_{\alpha}(\mathrm{f}(\mathrm{X}-\mathrm{Y}))$

$$
\begin{aligned}
& =\eta_{\alpha}(\mathrm{f}(\mathrm{X})-\mathrm{f}(\mathrm{Y})) \\
& \leq \max \left\{\eta_{\alpha}(\mathrm{f}(\mathrm{X})), \eta_{\alpha}(\mathrm{f}(\mathrm{Y}))\right\} \\
& =\max \left\{\left(\mathrm{f}^{-1}\left(\eta_{\alpha)}\right)(\mathrm{X}),\left(\mathrm{f}^{-1}\left(\eta_{\alpha}\right)\right)(\mathrm{Y})\right\}\right.
\end{aligned}
$$

Therefore, $\left(f^{-1}\left(\eta_{\alpha}\right)\right)(X-Y) \leq$ 


\section{International Journal of Science and Research (IJSR) \\ ISSN (Online): 2319-7064}

Index Copernicus Value (2013): 6.14 | Impact Factor (2015): 6.391

ii. $\begin{aligned}\left(\mathrm{f}^{-1}\left(\eta_{\alpha}\right)\right)(\mathrm{XY}) & =\eta_{\alpha}(\mathrm{f}(\mathrm{XY})) \\ = & \eta_{\alpha}(\mathrm{f}(\mathrm{X}) \mathrm{f}(\mathrm{Y})) \\ \leq & \max \left\{\eta_{\alpha}(\mathrm{f}(\mathrm{X})), \eta_{\alpha}(\mathrm{f}(\mathrm{Y}))\right\} \\ = & \max \left\{\left(\mathrm{f}^{-1}\left(\eta_{\alpha}\right)\right)(\mathrm{X}),\left(\mathrm{f}^{-1}\left(\eta_{\alpha}\right)\right)(\mathrm{Y})\right\}\end{aligned}$

Therefore, $\quad\left(f^{-1}\left(\eta_{\alpha}\right)\right)(X Y) \leq$

$$
\max \left\{\left(\mathrm{f}^{-1}\left(\eta_{\alpha}\right)\right)(\mathrm{X}),\left(\mathrm{f}^{-1}\left(\eta_{\alpha}\right)\right)(\mathrm{Y})\right\} .
$$

iii. $\left(\mathrm{f}^{-1}\left(\eta_{\alpha}\right)\right)(\mathrm{XYZ})=\eta_{\alpha}(\mathrm{f}(\mathrm{XYZ}))$

$$
\begin{aligned}
& =\eta_{\alpha}(f(X) f(Y) f(Z)) \\
& \leq \max \left\{\eta_{\alpha}(f(X)), \eta_{\alpha}(f(Z))\right\} \\
& =\max \left\{\left(f^{-1}\left(\eta_{\alpha}\right)\right)(X),\left(f^{-1}\left(\eta_{\alpha}\right)\right)(Z)\right\} \\
& =\max \left\{\left(f^{-1}\left(\eta_{\alpha}\right)\right)(X),\left(f^{-1}\left(\eta_{\alpha}\right)\right)(Z)\right\}
\end{aligned}
$$

$\left(\mathrm{f}^{-1}\left(\eta_{\alpha}\right)\right)(\mathrm{XYZ}) \leq \max \left\{\left(\mathrm{f}^{-1}\left(\eta_{\alpha}\right)\right)(\mathrm{X}),\left(\mathrm{f}^{-1}\left(\eta_{\alpha}\right)\right)(\mathrm{Z})\right\}$

Therefore, $f^{-1}\left(\eta_{\alpha}\right)$ is an anti-fuzzy HX bi- ideal of $\mathfrak{R}_{1}$.

\subsection{Theorem}

Let $\mathfrak{R}_{1}$ and $\mathfrak{R}_{2}$ be any two HX rings on the rings $\mathrm{R}_{1}$ and $\mathrm{R}_{2}$ respectively. Let $\mathrm{f}: \mathfrak{R}_{1} \rightarrow \mathfrak{R}_{2}$ be an anti homomorphism onto HX rings. Let $\lambda_{\mu}$ be an anti-fuzzy HX bi-ideal of $\Re_{1}$ then $\mathrm{f}$ ( $\lambda_{\mu}$ ) is an anti-fuzzy HX bi-ideal of $\mathfrak{R}_{2}$, if $\lambda_{\mu}$ has an infimum property and $\lambda_{\mu}$ is f-invariant.

\section{Proof}

Let $\mu$ be a fuzzy subset of $R_{1}$ and $\lambda_{\mu}$ is an anti-fuzzy $\mathrm{HX}$ biideal of $\mathfrak{R}_{1}$.

There exist $\mathrm{X}, \mathrm{Y}, \mathrm{Z} \in \mathfrak{R}_{1}$ such that $\mathrm{f}(\mathrm{X}), \mathrm{f}(\mathrm{Y}), \mathrm{f}(\mathrm{Z}) \in \mathfrak{R}_{2}$, i. $\left(f\left(\lambda_{\mu}\right)\right)(f(X)-f(Y))=\left(f\left(\lambda_{\mu}\right)\right)(f(Y-X))$,

$$
\begin{aligned}
& \quad=\lambda_{\mu}(\mathrm{Y}-\mathrm{X}) \\
& \leq \max \left\{\lambda_{\mu}(\mathrm{Y}), \lambda_{\mu}(\mathrm{X})\right\} \\
& \quad \leq \max \left\{\lambda_{\mu}(\mathrm{X}), \lambda_{\mu}(\mathrm{Y})\right\} \\
& =\max \left\{\left(\mathrm{f}\left(\lambda_{\mu}\right)\right)(\mathrm{f}(\mathrm{X})),\left(\mathrm{f}\left(\lambda_{\mu}\right)\right)(\mathrm{f}(\mathrm{Y}))\right\}
\end{aligned}
$$

Therefore, $\left(\mathrm{f}\left(\lambda_{\mu}\right)\right)(\mathrm{f}(\mathrm{X})-\mathrm{f}(\mathrm{Y})) \leq$

$$
\max \left\{\left(\mathrm{f}\left(\lambda_{\mu}\right)\right)(\mathrm{f}(\mathrm{X})),\left(\mathrm{f}\left(\lambda_{\mu}\right)\right)(\mathrm{f}(\mathrm{Y}))\right\} .
$$

ii. $\left(f\left(\lambda_{\mu}\right)\right)(f(X) f(Y))=\left(f\left(\lambda_{\mu}\right)\right)(f(Y X))$,

$$
=\lambda_{\mu}(\mathrm{YX})
$$$$
\leq \max \left\{\lambda_{\mu}(\mathrm{Y}), \lambda_{\mu}(\mathrm{X})\right\}
$$

$$
\leq \max \left\{\lambda_{\mu}(\mathrm{X}), \lambda_{\mu}(\mathrm{Y})\right\}
$$

$$
=\max \left\{\left(\mathrm{f}\left(\lambda_{\mu}\right)\right)(\mathrm{f}(\mathrm{X})),\left(\mathrm{f}\left(\lambda_{\mu}\right)\right)(\mathrm{f}(\mathrm{Y}))\right\}
$$

Therefore, $\left(\mathrm{f}\left(\lambda_{\mu}\right)\right)(\mathrm{f}(\mathrm{X}) \mathrm{f}(\mathrm{Y})) \leq$

$\max \left\{\left(\mathrm{f}\left(\lambda_{\mu}\right)\right)\left(\mathrm{f}(\mathrm{X}),\left(\mathrm{f}\left(\lambda_{\mu}\right)\right)(\mathrm{f}(\mathrm{Y}))\right\}\right.$.

iii. $\left(\mathrm{f}\left(\lambda_{\mu}\right)\right)(\mathrm{f}(\mathrm{X}) \mathrm{f}(\mathrm{Y}) \mathrm{f}(\mathrm{Z}))=\left(\mathrm{f}\left(\lambda_{\mu}\right)\right)(\mathrm{f}(\mathrm{ZYX}))$

$$
\begin{aligned}
& =\lambda_{\mu}(\mathrm{ZYX}) \\
& \leq \max \left\{\lambda_{\mu}(\mathrm{Z}), \lambda_{\mu}(\mathrm{X})\right\} \\
& \leq \max \left\{\lambda_{\mu}(\mathrm{X}), \lambda_{\mu}(\mathrm{Z})\right\} \\
& =\max \left\{\left(\mathrm{f}\left(\lambda_{\mu}\right)\right)(\mathrm{f}(\mathrm{X})),\left(\mathrm{f}\left(\lambda_{\mu}\right)\right)(\mathrm{f}(\mathrm{Z}))\right\}
\end{aligned}
$$

$\left(\mathrm{f}\left(\lambda_{\mu}\right)\right)(\mathrm{f}(\mathrm{X}) \mathrm{f}(\mathrm{Y}) \mathrm{f}(\mathrm{Z})) \leq \max \left\{\left(\mathrm{f}\left(\lambda_{\mu}\right)\right)(\mathrm{f}(\mathrm{X})),\left(\mathrm{f}\left(\lambda_{\mu}\right)\right)(\mathrm{f}(\mathrm{Z}))\right\}$

Hence, $\left(\mathrm{f}\left(\lambda_{\mu}\right)\right)$ is an anti-fuzzy HX bi- ideal on $\mathfrak{R}_{2}$.

\subsection{Theorem}

Let $\mathfrak{R}_{1}$ and $\mathfrak{R}_{2}$ be any two $\mathrm{HX}$ rings on $\mathrm{R}_{1}$ and $\mathrm{R}_{2}$ respectively. Let $\mathrm{f}: \mathfrak{R}_{1} \rightarrow \mathfrak{R}_{2}$ be an anti-homomorphism on $\mathrm{HX}$ rings. Let $\eta_{\alpha}$ be an anti-fuzzy $\mathrm{HX}$ bi-ideal of $\Re_{2}$ then $\mathrm{f}^{-1}$ $\left(\eta_{\alpha}\right)$ is an anti-fuzzy HX bi- ideal of $\mathfrak{R}_{1}$.

\section{Proof}

Let $\mu$ be a fuzzy subset of $R_{1}$ and $\eta_{\alpha}$ is an anti-fuzzy HX biideal of $\mathfrak{R}_{1}$.

There exist $X, Y, Z \in \mathfrak{R}_{1}$ such that $\mathrm{f}(\mathrm{X}), \mathrm{f}(\mathrm{Y}), \mathrm{f}(\mathrm{Z}) \in \mathfrak{R}_{2}$,

i. $\left(f^{-1}\left(\eta_{\alpha}\right)\right)(X-Y)=\eta_{\alpha}(f(X-Y))$

$$
\begin{aligned}
& =\eta_{\alpha}(\mathrm{f}(\mathrm{Y})-\mathrm{f}(\mathrm{X})) \\
& \leq \max \left\{\eta_{\alpha}(\mathrm{f}(\mathrm{Y})), \eta_{\alpha}(\mathrm{f}(\mathrm{X}))\right\} \\
& \leq \max \left\{\eta_{\alpha}(\mathrm{f}(\mathrm{X})), \eta_{\alpha}(\mathrm{f}(\mathrm{Y}))\right\} \\
& =\max \left\{\left(\mathrm{f}^{-1}\left(\eta_{\alpha)}\right)(\mathrm{X}),\left(\mathrm{f}^{-1}\left(\eta_{\alpha}\right)\right)(\mathrm{Y})\right\}\right.
\end{aligned}
$$

Therefore, $\left(f^{-1}\left(\eta_{\alpha}\right)\right)(X-Y) \leq$

$$
\text { ii. } \begin{aligned}
\left(\mathrm{f}^{-1}\left(\eta_{\alpha}\right)\right)(\mathrm{XY}) & =\max \left\{\left(\mathrm{f}^{-1}\left(\eta_{\alpha}\right)\right)(\mathrm{X}),\left(\mathrm{f}^{-1}\left(\eta_{\alpha}\right)\right)(\mathrm{Y})\right\} \\
& =\eta_{\alpha}(\mathrm{f}(\mathrm{XY})) \\
& \leq \max \{\mathrm{f}(\mathrm{X})) \\
& \leq \max \left\{\eta_{\alpha}\left(\mathrm{f}(\mathrm{Y}(\mathrm{X})), \eta_{\alpha}(\mathrm{f}(\mathrm{X}))\right\}\right. \\
& =\max \left\{\left(\mathrm{f}^{-1}\left(\eta_{\alpha}\right)\right)(\mathrm{X}),\left(\mathrm{f}^{-1}\left(\eta_{\alpha}\right)\right)(\mathrm{Y})\right\}
\end{aligned}
$$

Therefore, $\left(f^{-1}\left(\eta_{\alpha}\right)\right)(X Y) \leq$

$$
\max \left\{\left(\mathrm{f}^{-1}\left(\eta_{\alpha}\right)\right)(\mathrm{X}),\left(\mathrm{f}^{-1}\left(\eta_{\alpha}\right)\right)(\mathrm{Y})\right\} .
$$

iii. $\left(\mathrm{f}^{-1}\left(\eta_{\alpha}\right)\right)(\mathrm{XYZ})=\eta_{\alpha}(\mathrm{f}(\mathrm{XYZ}))$

$$
=\eta_{\alpha}(\mathrm{f}(\mathrm{Z}) \mathrm{f}(\mathrm{Y}) \mathrm{f}(\mathrm{X}))
$$$$
\leq \max \left\{\eta_{\alpha}(\mathrm{f}(\mathrm{Z})), \eta_{\alpha}(\mathrm{f}(\mathrm{X}))\right\}
$$$$
\leq \max \left\{\eta_{\alpha}(\mathrm{f}(\mathrm{X})), \eta_{\alpha}(\mathrm{f}(\mathrm{Z}))\right\}
$$$$
=\max \left\{\left(\mathrm{f}^{-1}\left(\eta_{\alpha}\right)\right)(\mathrm{X}),\left(\mathrm{f}^{-1}\left(\eta_{\alpha}\right)\right)(\mathrm{Z})\right\}
$$$$
=\max \left\{\left(\mathrm{f}^{-1}\left(\eta_{\alpha}\right)\right)(\mathrm{X}),\left(\mathrm{f}^{-1}\left(\eta_{\alpha}\right)\right)(\mathrm{Z})\right\}
$$

$\left(\mathrm{f}^{-1}\left(\eta_{\alpha}\right)\right)(\mathrm{XYZ}) \leq \max \left\{\left(\mathrm{f}^{-1}\left(\eta_{\alpha}\right)\right)(\mathrm{X}),\left(\mathrm{f}^{-1}\left(\eta_{\alpha}\right)\right)(\mathrm{Z})\right\}$

Therefore, $\mathrm{f}^{-1}\left(\eta_{\alpha}\right)$ is an anti-fuzzy HX bi- ideal of $\mathfrak{R}_{1}$.

\section{References}

[1] Bing-xueYao and Yubin-Zhong, The construction of power ring, Fuzzy information and Engineering (ICFIE), ASC 40, pp.181-187, 2007.

[2] Bing-xueYao and Yubin-Zhong, Upgrade of algebraic structure of ring, Fuzzy information and Engineering 2:219-228(2 009).

[3] Dheena.P and Mohanraaj.G, T- fuzzy ideals in rings, International Journal of computational cognition, volume 9, No.2, 98-101, June 2011.

[4] Jianming Zhan, Yunqiang Yin, Yang Xu, A new view of fuzzy ideals in rings, Anals of fuzzy mathematics \& Informatics, Volume 2, No.1(July 2011), pp.99-115.

[5] Kuroki.N On fuzzy ideals and fuzzy bi-ideals in semi groups, Fuzzy sets and systems 5(1981), 203-215.

[6] Lajos.S, Szasz, Bi-ideals in associative rings, Acta Sci.Math., 32(1971), 185-193.

[7] Li Hong Xing, HX group, BUSEFAL, 33(4), 31-37, October 1987.

[8] Li Hong Xing, HX ring, BUSEFAL, 34(1) 3-8, January 1988.

[9] Liu. W.J., Fuzzy invariant subgroups and fuzzy ideals, Fuzzy sets and systems, 8:133-139.

[10] Mashinchi.M and Zahedi.M.M, On fuzzy ideals of a ring, J.Sci.I.R.Iran, 1(3), 208- 210(1990)

[11] Mukherjee.T.K \& Sen.M.K., On fuzzy ideals in rings, Fuzzy sets and systems, 21, 99-104, 1987.

[12] Muthuraj.R, Ramila Gandhi.N, Homomorphism and anti homomorphism of fuzzy HXideals of a HX ring, Discovery, Volume 21, Number 65, July 1, 2014, 2024. 
[13] Rosenfeld. A., Fuzzy Groups, Journal of mathematical analysis and applications, 35(1971), 512-517.

[14] Sanjib Kumar Datta, On the lower level sets of anti fuzzy bi-ideals in rings, International Journal of Pure and Applied Mathematics, Volume 51 No.3 2009, 359-362.

[15] Sanjib Kumar Datta, On anti fuzzy bi-ideals in rings, International Journal of Pure and Applied Mathematics, Volume 51 No.3 2009, 375-382.

[16]Zadeh. L. A., Fuzzy sets, Information and control, 8, (1965) 338-353. 